\title{
Frequency Detection of Single channel Steady State Visual Evoked Potential using Canonical correlation Analysis
}

\author{
Mukesh Kumar Ojha, Manoj Kumar Mukul, Ravinder Nath Rajotia, Nitin Tyagi
}

\begin{abstract}
Wave generated into visual cortex of brain, when subject focused his/her attention on visual stimulus flickers at certain frequency. The main challenge with SSVEP Based Brain computer interface (BCI) System is to detect the stimulus frequency from recorded brain signal. Canonical Correlation analysis $(C C A)$ is one of the most popular methods to recognize the frequency of Steady state visual evoked potential (SSVEP). This paper focuses on the study of CCA algorithm to recognize the SSVEP signal frequency. For experiment purpose, a single channel data with flickering frequency in the range of $(6 \mathrm{~Hz}-10 \mathrm{~Hz})$ is used. The performance of the BCI System is measured in terms of detection accuracy and Information transmission rate (ITR). The maximum accuracy is obtained as $83.90 \%$ and ITR is 15.35 at stimulus frequency of $8.2 \mathrm{~Hz}$
\end{abstract}

Index Terms: Steady State Visual evoked potential (SSVEP); Brain computer interface (BCI); PSDA, Electroencephalogram (EEG); Canonical correlation analysis (CCA);

\section{INTRODUCTION}

$\mathrm{BCI}$ is a non-invasive direct communication system by which user's intention is directly conveyed to external environment without involving the normal output pathways of peripheral nervous and muscles. In other words, BCIs main aim is in establishing the link between user and external world [1].In non-invasive BCIs, electroencephalography (EEG) is commonly used because of high resolution, ease of acquisition and cost effectiveness as compare to other brain monitoring modalities. In past few decades many BCIs System have been investigated that include P300, motor imagery, Steady state visual evoked potential (SSVEP). Among these, SSVEP is more popular and widely acceptable because of high information transmission rate (ITR), minimal training time and high signal to noise ratio [7],[9].

SSVEP is the brain's electrical response to repetitive visual stimulation that can be recorded from the scalp over the visual cortex with maximum amplitude occurs at occipital region[1],[7].The basic SSVEP based BCI system offer several flickering visual stimuli with different frequencies as
Target. When candidate is asked to focus on one of the target, the response with specific spectrum component can be identified. Thus the target, the candidate is focusing on will be determined. With this phenomenon, SSVEP Based BCI System is developed to generate a command to control external Machine by recognizing the SSVEP frequency component in EEG Signal. Since SSVEP signal is contaminated by other EEG activity and Background noise in to Brain. Thus How to recognize the frequency component of SSVEP Signal from recorded EEG Signal with better accuracy and high information transmission rate is the challenging task to design SSVEP Based BCI System.

The most wide spread signal processing technique to extract the SSVEP Frequency components from the acquired EEG Signal is based on Power spectral density Analysis (PSDA) using FFT of a sliding data window with a fixed length .In past few years several approaches have been proposed to improve the robustness upon FFT based Spectral analysis method. . Canonical Correlation Analysis (CCA) is another efficient and widely used technique to recognize the SSVEP frequency component from acquired EEG Signal [4]. CCA is a multivariate statistical approach that estimates the degree of correlation between with two multivariate signals. The largest correlation between recorded EEG signal and Pre-constructed reference signal is used to identify the target. The reference signal is the series of sine-cosine wave consists of fundamental and harmonic frequency of SSVEP Stimulus. The stimulus frequency with maximum correlation coefficient is considered as target frequency

The rest of this paper is organized as follows. Section 2 deals with the overview on SSVEP Based BCI data acquisition system. Section3 explain the method applied to extract the feature of SSVEP Signal. Section 4 present the result of applied method Section 5 finally concludes the paper.
Revised Manuscript Received on October 22, 2019.

Mukesh Kumar ojha, Department of Electronics \& Communication,Birla institute of Technology, Mesra,Ranchi, Jharkhan Manoj Kumar Mukul , Department of Electronics \& Communication,Birla institute of Technology, Mesra,Ranchi, Jharkhan Ravinder Nath Rajotia, Department of Electronics \& Communication, JIMS Engg. Mgmt. Tech Campus, Gr. Noida, UP

Nitin Tyagi, Department of Electronics \& Communication, JIMS Engg. Mgmt. Tech Campus, Gr. Noida, UP * $\&$ ,

. 


\section{OVERVIEW OF SSVEP BASED BCI DATA ACQUISITION SYSTEM}

\section{A. SSVEP Based BCI System}

The block diagram of SSVEP Based BCI System is shown in figure-1.The entire SSVEP-Based BCI module consists of EEG acquisition system, Signal processing (frequency detection) algorithm and device control method. SSVEP based BCI System is operated by showing number of visual light flickering at specific frequency on the monitor of screen. When subject asked to focus on specific stimulus, the corresponding stimulus frequency dominantly appears into spectral representation of EEG Signal.

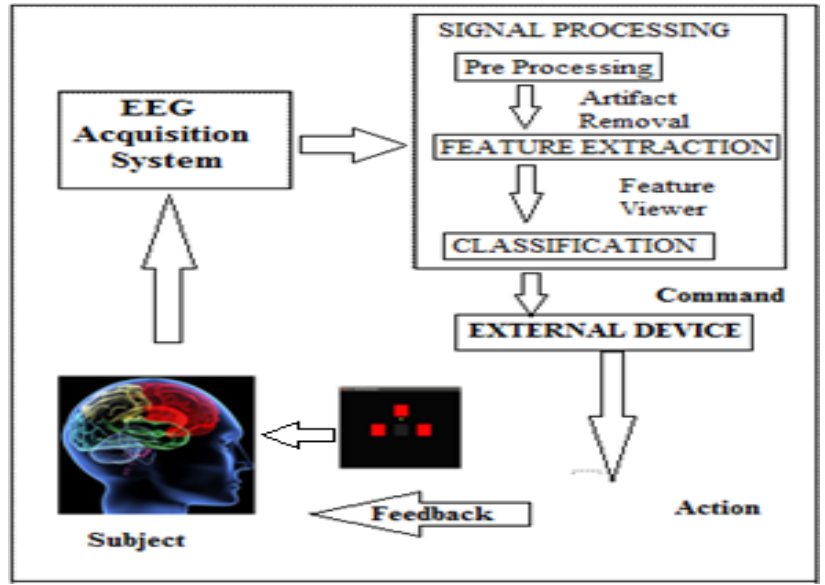

Figure 1: SSVEP based BCI System

The function of signal processing unit is used to detect the presence of SSVEP Signal into recorded EEG Signal and to determine the target frequency. Finally, each target frequency is classified to generate a unique command using classifier method. BCI System uses this unique command to control the external device such as cursor movement, wheel chair movement.

\section{B. Data Description}

A single channel offline data set recorded at sampling frequency of $512 \mathrm{~Hz}$ is used in this paper[14].A 10-20 electrode placement system is used to acquire EEG Signal by placing the electrode at $\mathrm{Oz}$ position on the scalp of brain. The Stimulus frequency range is $6 \mathrm{~Hz}-10 \mathrm{~Hz}$. The recorded EEG Signal when candidate focused on stimulus frequency of $10 \mathrm{~Hz}$ is shown in figure-2.

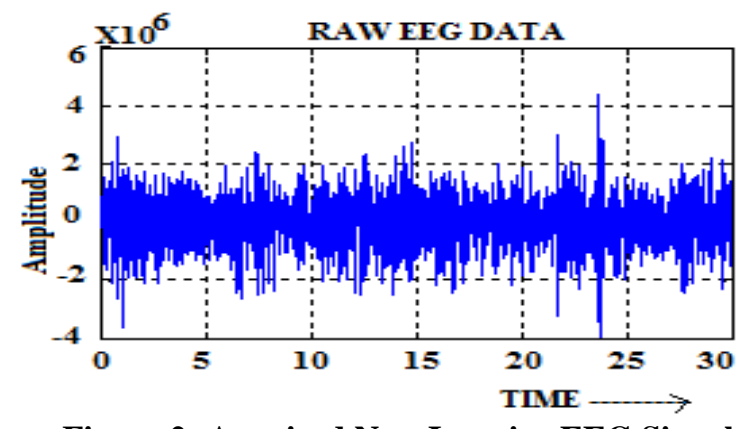

Figure 2: Acquired Non-Invasive EEG Signal

\section{CANONICAL CORRELATION ANALYSIS (CCA) METHOD}

Canonical correlation analysis is a multivariate statistical approach with the aim to find the correlation between two sets of data[4]. Its main strength CCA algorithm is to transform two sets of data says $\mathrm{X}$ and $\mathrm{Y}$ to a new sets of variable $\bar{x}=W_{x} X^{T}$ and $\bar{y}=W_{y} Y^{T}$; where $W_{x}$ and $W_{y}$ the canonical variants. The function of CCA Algorithm is to find the weight vector $W_{x}$ and $W_{y}$ in such a way that correlation between $\bar{x}$ and $\bar{y}$ is maximized. Mathematically this is given as;

$$
\begin{gathered}
\rho=\max \left(W_{x^{s}} W_{y}\right) \frac{E\left[x y^{T}\right]}{\sqrt{E\left[x x^{T}\right] E\left[y y^{T}\right]}} \\
\rho=\max \left(W_{x^{v}} W_{y}\right) \frac{W_{x} \zeta_{X Y} W_{y}}{\sqrt{W_{x} C_{X X} W_{x}{ }^{T} W_{y} C_{Y Y} W_{y}{ }^{T}}}
\end{gathered}
$$

Where $\rho$ denote the correlation coefficient between data sets $\mathrm{X}$ and $\mathrm{Y}$ that can be maximized by maximizing $\mathrm{W}_{\mathrm{x}}$ and $\mathrm{W}_{\mathrm{Y}}$. According to this approach the extracted correlation coefficient for all the stimulus frequency, the maximum correlation coefficient is considered as SSVEP frequency. The mathematical approach to detect single or multi channel SSVEP signal using CCA technique is discussed here Assume there are $\mathrm{K}$ target frequency to be recognized into SSVEP based BCI System. Let X is the recorded EEG Signal from $i_{t h}$ channel with n number of sample in each channel. Assume $\boldsymbol{Y}_{\boldsymbol{k}}$ is the reference signal at $\mathbf{k}^{\text {th }}$ stimulus frequency $(\mathrm{k}=1,2 \ldots . \mathrm{k})$ consists of sine-cosine function given as

$$
Y_{k}=\left[\begin{array}{c}
\sin \left(2 \pi f_{k} t\right) \\
\cos \left(2 \pi f_{k} t\right) \\
\cdot \\
\cdot \\
\sin \left(2 \pi N_{H} f_{k} t\right) \\
\cos \left(2 \pi N_{H} f_{k} t\right)
\end{array}\right], t=\frac{1}{f_{s}}, \frac{2}{f_{s}}, \ldots . . \frac{k}{f_{s}}
$$

Where $\mathrm{N}_{\mathrm{H}}$ denote the number of harmonics and is the sampling frequency. The aim of CCA method is to find the correlation between recorded signal $\mathrm{X}$ and each reference signal using equation (1). The SSVEP target frequency is recognized as $\mathrm{f}_{\mathrm{t}}=\max _{\mathrm{f}_{\mathrm{k}}} \mathrm{p}_{\mathrm{k}}, \mathrm{k}=1,2, \ldots \ldots \mathrm{k}$.

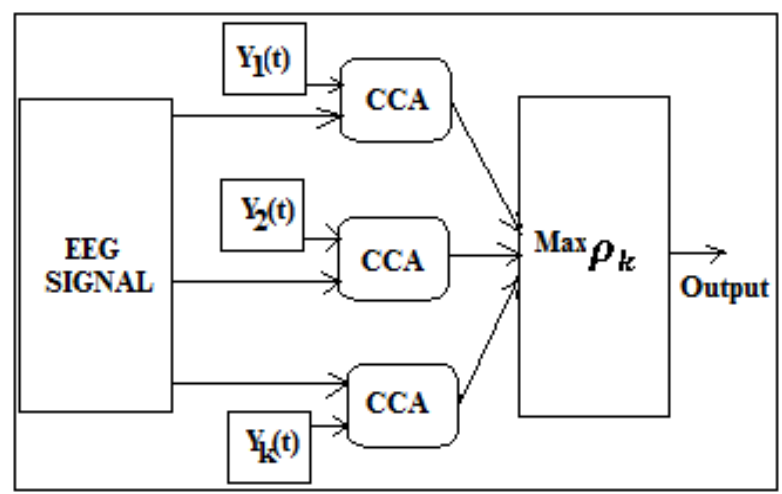

Figure 3: SSVEP Frequency detection using CCA 


\section{RESULT AND DISCUSSION}

\section{A. Canonical Correlation Analysis}

Table 1: Correlation Coefficient trails at different target frequency for subject-4 using CCA

\begin{tabular}{|l|l|l|l|l|}
\hline $\begin{array}{l}\text { Subject- } \\
4\end{array}$ & $\begin{array}{l}\text { Correlation } \\
\text { coefficient } \\
\text { at Target } \\
\text { Frequency= } \\
10 \mathrm{~Hz}\end{array}$ & $\begin{array}{l}\text { Correlation } \\
\text { coefficient } \\
\text { at Target } \\
\text { Frequency= } \\
6 \mathrm{~Hz}\end{array}$ & $\begin{array}{l}\text { Correlation } \\
\text { coefficient } \\
\text { at Target } \\
\text { Frequency= } \\
7 \mathrm{~Hz}\end{array}$ & $\begin{array}{l}\text { Correlation } \\
\text { coefficient } \\
\text { at Target } \\
\text { Frequency= } \\
8.2 \mathrm{~Hz}\end{array}$ \\
\hline Trail-1 & 0.4214 & 0.0835 & 0.0686 & 0.0713 \\
\hline Trail-2 & 0.1270 & 0.0263 & 0.0459 & 0.0222 \\
\hline Trail-3 & 0.2721 & 0.0480 & 0.0153 & 0.0461 \\
\hline Trail-4 & 0.0702 & 0.3644 & 0.0881 & 0.0048 \\
\hline Trail-5 & 0.0166 & 0.1555 & 0.0419 & 0.0453 \\
\hline Trail-6 & 0.0472 & 0.0177 & 0.0292 & 0.0522 \\
\hline Trail-7 & 0.0568 & 0.1104 & 0.3799 & 0.0601 \\
\hline Trail-8 & 0.0207 & 0.0087 & 0.2999 & 0.0018 \\
\hline Trail-9 & 0.0409 & 0.0325 & 0.0629 & 0.0259 \\
\hline Trail-10 & 0.0723 & 0.0639 & 0.0108 & 0.4596 \\
\hline Trail-11 & 0.0396 & 0.0220 & 0.0438 & 0.0319 \\
\hline Trail-12 & 0.0249 & 0.0157 & 0.0538 & 0.0770 \\
\hline
\end{tabular}
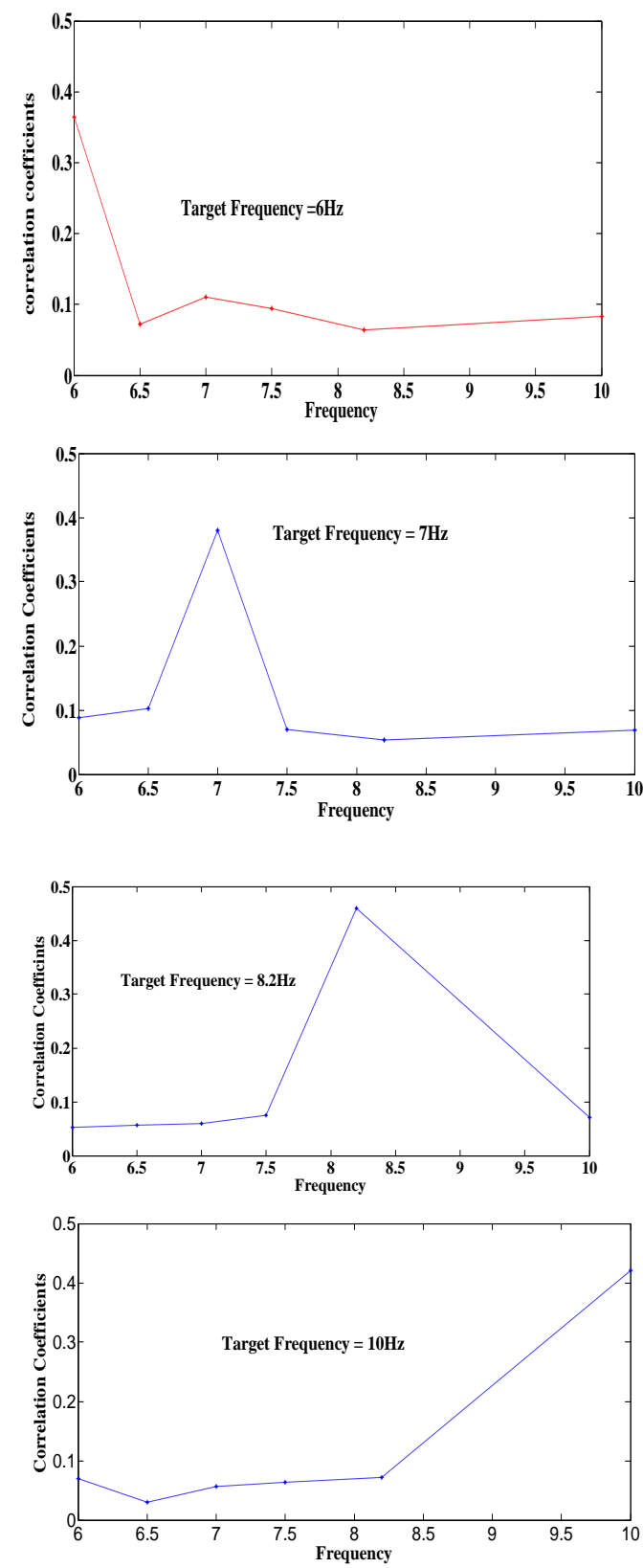

Figure 4: Max Correlation coefficient of SSVEP Signal occur at reference frequency
The correlation coefficient obtained using CCA algorithm in different trails for subject-1 is shown in table-1. The EEG Signal recorded at stimulus frequency $10 \mathrm{~Hz}$ in trials [1-3], at $6 \mathrm{~Hz}$ in trials [4-6], at stimulus frequency $7 \mathrm{~Hz}$ in trials [7-9], at stimulus frequency $8.2 \mathrm{~Hz}$ in trials [10-12].In Fig-4, the correlation coefficients is obtained between the recorded signal and the reference signal (Sine-cosine of certain frequency). From figure-4 we can conclude that SSVEP Signal of frequency same as the reference signal frequency have the highest correlation coefficient compared to SSVEP Signal whose frequency is different from the reference signal frequency. Higher the value of correlation coefficient at reference signal frequency indicates the maximum similarity between SSVEP Signal and the reference signal

\section{B. Performance Evaluation:}

The most common measure to evaluate the performance of SSVEP Based BCI is accuracy and Information transmission rate (ITR). In this paper accuracy of the system is measured in terms Maximum correlation coefficients obtained at target frequency. Mathematically this is given by;

$$
\text { Accuracy }=\frac{\max \left(\rho_{f}\right)}{\sum_{k=1}^{M} \max \left(\rho_{f_{K}}\right)}
$$

Where $\rho_{\mathrm{f}}$ is the maximum correlation coefficients at Target Frequency, $M$ is the number of Stimulus frequency. The Information transmission rate (ITR) [9] is defined by equation

$$
B_{t}=\log _{2} N+P \log _{2} P+(1-P) l_{0}
$$

Where $\mathrm{N}$ is the number of Stimulus, $\mathrm{P}$ indicates the classification accuracy and indicates the Bit per Target. The information transmission rate in Bits per minute is given by [8], [9];

$$
B_{m}=\frac{60}{T} C_{N^{*}} B_{t}
$$

Where $\mathbf{C}_{\mathbf{N}}$ is the number of classification, $\mathbf{T}$ is the minimal time between two consecutive command classifications. The table -2 indicates the Accuracy and ITR obtained for each target frequency of subject-1.

Table: 2 Detection accuracy (\%) and ITR (Bits/Minute) for each Stimulation frequency of Subject-1

\begin{tabular}{|c|c|c|}
\hline $\begin{array}{c}\text { Stimulus } \\
\text { Frequency }\end{array}$ & $\begin{array}{c}\text { Accuracy } \\
\text { (\%age) }\end{array}$ & $\begin{array}{c}\text { ITR } \\
\text { (Bits/min) }\end{array}$ \\
\hline $6 \mathrm{~Hz}$ & $\mathbf{8 1 . 2 0}$ & 13.46 \\
\hline $7 \mathrm{~Hz}$ & $\mathbf{8 1 . 6 0}$ & 13.34 \\
\hline $8.2 \mathrm{~Hz}$ & $\mathbf{8 3 . 9 0}$ & 15.35 \\
\hline $10 \mathrm{~Hz}$ & $\mathbf{7 8 . 5 0}$ & 12.78 \\
\hline
\end{tabular}

The table-2 lists the detection accuracy for each stimulation frequency and their corresponding Information transmission rate (ITR). The above result shows that detection accuracy is increasing in low frequency range $(6 \mathrm{~Hz}-8.2 \mathrm{~Hz})$ of SSVEP Signal while start decreasing in high frequency range (above $8.2 \mathrm{~Hz}$ ) of SSVEP Signal.

\section{CONCLUSION AND FUTURE WORK}

The main concern of this paper is the study of a novel method based on canonical correlation analysis to detect SSVEP Frequency from single channel EEG Signal. This method uses the pre-constructed sine-cosine signal as reference signal for SSVEP Frequency recognition. 
The result indicates that the detection accuracy is increasing in low frequency range $(6 \mathrm{~Hz}-8 \mathrm{~Hz})$ and start decreasing in High frequency range (above $8.2 \mathrm{~Hz}$ ). The future work will be focused on development of a mechanism by which detection accuracy of SSVEP Signal can be improved into high frequency range. The other main concern in our future work will be to apply our proposed method to the implementation of multi-command, real time portable BCI System.

\section{RFERENECES}

1. Y. Wang, X.Gao, B. Hong, C.Jia and S.Gao, "Brain-Computer Interfaces Based on Visual Evoked Potentials", IEEEEng.Med.Biol.Magazine, vol. 27, no.5 pp.64-71,2008.

2. M.Cheng, X. Gao, S.Gao, and D.Xu "Design and implementation of a brain-computer interface with high transfer rates, "IEEETrans. Biomed. Eng. Vol.49 no.10pp.1181-1186,2002.

3. G. Bin, X.Gao, Z.Yan, B. Hong, and S.Gao, "An online multichannel SSVEP-based brain-computer interface using a canonical correlation analysis method." J. Neural Eng. Vol.6, no4, 2009.

4. Z. Lin, C.Zhang, W.Wu, and X.Gao, "Frequency recognition based on canonical correlation analysis for SSVEP-based BCIs," IEEE Trans. Biomed.Eng., vol.54 no.6 pp. 1172-1176, 2007.

5. Chun-Shu Wei, Yuan-pin Lin T.P.Jung, “ Detection of Steady state visual evoked potential using differential Canonical correlation Analysis," 6th Annual Int. IEEE EMBS Conf. Neural Eng. Pp 57-60,2013.

6. B.Z.Allison, D.J. McFarland, G.Schalk, S.Zheng, M.Jackson and J.R Wolpaw "Towards an independent brain computer interface using steady state visual evoked potentials," Clinical Neurophysiology, pp 399-408, 2008.

7. Y.Zhang, G.Zhou, J.Jin, M.Wang, X.Wang, A.Cichoki, “ L1-regularized multiway canonical correlation analysis for SSVEP-based BCI, IEEE Trans,Neural Syst. Rehabilitation Eng. PP 887-896,2013.

8. O.Friman, I. Volosyak and A.Graser"Multichannel detection of steady-state visual evoked potentials for brain-computer interfaces, IEEE Trans. Biomed. Eng. PP 742-750, 2007.

9. Ivan Volosyak "SSVEP based Bremen-BCI interface boosting information transfer rate" Journal of Neural Engineering, 2011, PP 702-709.

10. Ivan volosyak,tatsina Malechka, Diana Valbuena and Axel Graser " A Novel calibration Method for SSVEP based brain computer interfaces" European Signal Processing conference,2010.

11. Young Punsawad, yadchanan wangsawat " Multi command SSVEP-based BCI System via Single flickering frequency Half field stimulation pattern" IEEE EMBS Conference, PP 1101-1104, September, 2011

12. Zheng P. and Xiaorang Gao "Fixed point CCA Algorithm applied to SSVEP Based BCI System" 2013 IEEE Symposium on Computational intelligence Cognitive algorithm and Brain,2013.

13. Masaki Nakanishi, Yijun wang, yu-Te wang, Yasue mitsukusa “ Enhancing unsupervised canonical correlation analysis based frequency detection of SSVEP by incorporating background EEG", $36^{\mathrm{TH}}$ Annual International conference of the IEEE Engineering in medicin and Biology Society 2014.

14. Vilic, A. The AVI SSVEP DATASET available online.

\section{AUTHORS PROFILE}

Mukesh Kumar ojha, Department of Electronics \& Communication,Birla institute of Technology, Mesra,Ranchi, Jharkhan Manoj Kumar Mukul, Department of Electronics \& Communication,Birla institute of Technology, Mesra,Ranchi, Jharkhan

Ravinder Nath Rajotia, Department of Electronics \& Communication, JIMS Engg. Mgmt. Tech Campus, Gr. Noida, UP

Nitin Tyagi, Department of Electronics \& Communication, JIMS

Engg. Mgmt. Tech Campus, Gr. Noida, UP 